Cahiers $d u$ MONDE RUSSE

\section{Cahiers du monde russe}

Russie - Empire russe - Union soviétique et États indépendants

$59 / 4 \mid 2018$

Varia

\title{
Biologie, médecine et santé au miroir de leur internationalisation
}

\section{Gregory Dufaud}

\section{OpenEdition}

\section{Journals}

Édition électronique

URL : https://journals.openedition.org/monderusse/10809

DOI : 10.4000/monderusse. 10809

ISSN : $1777-5388$

\section{Éditeur}

Éditions de l'EHESS

\section{Édition imprimée}

Date de publication : 1 octobre 2018

Pagination : 684-686

ISBN : 978-2-7132-2747-9

ISSN : $1252-6576$

\section{Référence électronique}

Gregory Dufaud, «Biologie, médecine et santé au miroir de leur internationalisation », Cahiers du monde russe [En ligne], 59/4 | 2018, mis en ligne le 01 octobre 2018, consulté le 12 janvier 2022. URL http://journals.openedition.org/monderusse/10809; DOI : https://doi.org/10.4000/monderusse. 10809 


\title{
Biologie, médecine et santé au miroir de leur internationalisation
}

Jean-Claude DUPONT, Jean-Gaël BARBARA, Eduard KOLCHINSKY, Marina LOSKUTOVA, dir.

\author{
Biologie et médecine en France et en Russie \\ Histoires croisées (fin $\mathrm{XVIII}^{\mathrm{e}}-\mathrm{XX}^{\mathrm{e}}$ siècle) \\ Paris : Hermann, 2016, 241 p.
}

Susan GRANT, dir.,

\section{Russian and Soviet Health Care from an International Perspective Comparing Professions, Practice and Gender, 1880-1960}

Basingstoke : Springer, 2017, $281 \mathrm{p}$.

Dans les années quatre-vingt-dix, l'avènement d'une « nouvelle histoire des sciences » s'est traduit par l'ouverture de chantiers de recherche sur les lieux de production savante, les savoirs et savoir-faire, et leur internationalisation ${ }^{1}$. C'est cette dernière voie que suivent ces deux recueils sur les sciences de la vie, la médecine et la santé publique en Russie. Leurs objectifs diffèrent quelque peu : faire une histoire croisée des échanges scientifiques entre la France et la Russie pour le volume de Jean-Claude Dupont et al., et situer l'expérience russe dans un contexte plus général pour celui de Susan Grant. En privilégiant le temps long, tous deux ont néanmoins en commun de venir interroger la spécificité de l'histoire de la Russie et cette fameuse « voie particulière » (Sonderweg) avancée par nombre d'intellectuels depuis maintenant deux siècles.

Le recueil sur la biologie et la médecine porte sur les relations scientifiques entre la France et la Russie de la fin du XVIII ${ }^{\mathrm{e}}$ siècle au $\mathrm{XX}^{\mathrm{e}}$ siècle, période pendant laquelle les échanges ont été permanents. Dans l'introduction, les auteurs convoquent la notion de « transfert culturel » qui renvoie au déplacement d'un objet d'un contexte culturel à un autre et aux appropriations opérées selon les conjonctures ${ }^{2}$. À travers le cas franco-russe, ils disent vouloir « souligner [...] la diversité, l'importance et les caractéristiques multilatérales d'interactions à l'intérieur d'un espace géographique et historique commun, celui de l'Europe des sciences » (p. 7). Pour ce faire, le recueil présente une collection de dix-neuf articles placés suivant un ordre grosso modo chronologique et divisés en deux parties, avec la première consacrée aux sciences naturelles et la seconde, à la physiologie et à la médecine. Si le grand nombre de textes fait la richesse de ce recueil, leur brièveté (à peine 4 pages pour celui sur le médecin Mottel, dit Michel, Kourilsky par Natalia Fedounina) suscite quelques frustrations. Par-delà les effets de juxtaposition et d'éclatement inhérents à ce genre $\mathrm{d}$ 'entreprise éditoriale, ce volume éclaire des phénomènes qui, en retour, suggèrent que le concept de transfert culturel n'est peut-être pas le plus à même de rendre compte des échanges entre la France et la Russie. Car s'il montre la diversité des lieux de fabrication de la science, les différentes formes de coopération scientifique, la variété des acteurs impliqués et les réceptions, il fait aussi apparaître que personnes et savoirs passent entre la France et la Russie via par exemple 
l'Allemagne et les pays Baltes. Ainsi du médecin et naturaliste Ludwig Heinrich Bojanus (1776-1827) qui, né en Alsace, a étudié à Darmstadt et à Iéna avant d'obtenir la chaire de médecine vétérinaire de l'université de Vilnius puis d'en devenir le recteur. Stéphane Schmitt insiste sur les liens maintenus par Bojanus avec ses collègues allemands. Ainsi du développement de la physiologie russe qui doit autant aux travaux des physiologistes français qu'à ceux des physiologistes allemands. Vladimir Samoilov rappelle d'ailleurs que si Ivan Pavlov (1849-1936) a appris le français pour lire les écrits de Claude Bernard en langue originale, c'est auprès de Karl Ludwing et Rudolph Heidenhain qu'il a parfait sa formation. Pour rendre compte de la complexité des interactions telle qu'exposée par l'ensemble des articles, la notion de « circulations transnationales » paraît plus adaptée, car insistant davantage sur la dimension multidirectionnelle des échanges et le caractère multisitué des appropriations ${ }^{3}$.

Cette approche par les circulations se retrouve dans le livre édité par Susan Grant où elle n'est néanmoins qu'un des aspects abordés. L'idée est de replacer l'expérience russe et soviétique en matières médicale et sanitaire dans une perspective internationale. Sur les onze articles du volume, quatre ne portent pas sur la Russie sans que l'on comprenne vraiment le principe ayant dicté le choix de ces derniers, sinon une logique d'opportunité dont Susan Grant tire le meilleur pour rédiger une introduction substantielle justifiant les grandes lignes directrices du recueil. La première partie de l'ouvrage, la plus cohérente, est consacrée aux « Professions et pratiques ». Les articles soulèvent la question des statuts, de la délimitation des domaines de compétences ou des formes de reconnaissance. Ils ont en commun de traiter des interactions entre des acteurs aux attentes variées et du travail effectué par ces derniers pour les réaliser : Kim Friedlander traite du processus de spécialisation médicale des années 1880 aux années 1920, Susan Grant de la formation des infirmières entre 1936 et 1941, Donald Filtzer de la médecine (mediko-sanitarnaja čast ${ }^{\prime}$ ) dans l'industrie de la défense pendant la Seconde Guerre mondiale et Benjamin Zajicek de la hiérarchie professionnelle entre 1932 et 1951 à travers le cas de la psychiatrie. La deuxième partie a trait au genre. Le seul article sur la Russie porte sur la féminisation de la profession médicale qui, intervenue dès la fin du XIX $\mathrm{X}^{\mathrm{e}}$ siècle, s'est poursuivie à l'époque soviétique où les femmes représentaient les trois quarts du personnel médical après la Seconde Guerre mondiale. Michelle DenBeste étudie ainsi, pour les années 1880-1905, les raisons ayant poussé les femmes à rejoindre les professions médicales et leurs engagements subséquents : hygiène, santé publique ou instruction féminine. Laura Kelly traite, elle, de l'Irlande entre 1880 et 1920 : elle avance que les femmes y ont bénéficié d'un réel soutien dans les milieux médicaux, à la différence de leurs collègues britanniques et américaines. Quant à Julie Fairman, Hafeeza Anchrum et Taryn Pochon, elles défendent une démarche « intersectionnelle » qui veut saisir, pour la profession d'infirmière, la nature des rapports de pouvoir entre femmes et hommes, et dénaturaliser les dynamiques de toutes sortes les définissant. La dernière partie s'intéresse aux connexions internationales et s'attache à une histoire croisée de la médecine. Les textes révèlent la mobilité du personnel médical par-delà les frontières, nous permettant de retrouver 
ici ce que le recueil de Jean-Claude Dupont et al. souligne aussi très bien. Comme dans la partie précédente, un seul texte est centré sur la Russie. Susan G. Solomon évoque les délégations du commissariat à la Santé en France et Allemagne dans les années vingt, dont l'objectif était à la fois de collecter des informations et de promouvoir les réalisations soviétiques. Elle présente les trajectoires différenciées des délégations et suggère les difficultés de Moscou à se faire une idée précise des situations locales et des conditions de travail de leurs représentants, d'où, selon elle, l'impossibilité à construire un bilatéralisme fort. Dans son chapitre, Jaime Lapeyre analyse la formation des infirmières en santé publique en Amérique du Nord durant l'entre-deux-guerres et l'action d'institutions qui, par exemple au sein de la fondation Rockfeller, s'emploient à l'encourager tant au niveau national qu'à l'échelle internationale. En raison des appropriations locales, la volonté de standardisation a toutefois produit des histoires nationales distinctes. Paul Weindling s'intéresse aux infirmières qui s'exilent en Grande-Bretagne entre 1933 et 1946: les 5831 réfugiés exerçant une profession médicale comptaient 667 infirmières. Alors même que le besoin en personnel infirmier était très grand, elles ont généralement rencontré de grandes difficultés à obtenir un permis de travail. Plusieurs d'entre elles sont néanmoins parvenues à devenir des figures éminentes au sein de la profession.

Ces deux ouvrages proposent finalement une histoire de la médecine et de la santé publique en Russie où l'étude des échanges se combine à une approche comparative. Cette manière de « déspécifier »l'histoire russe ne cherche pas à nier ses particularités, mais bien plutôt d'essayer d'identifier ses spécificités et ce qui les explique. Pour Susan Grant, la trajectoire de la médecine et de la santé publique russes à partir de la fin du $\mathrm{XIX}^{\mathrm{e}}$ siècle « ne réside pas dans la médecine ou la science per se, mais dans l'identité professionnelle, le rôle des acteurs de la santé et la structure du système de santé ». Et d'ajouter que la « révolution d'Octobre et l'opposition idéologique soviétique à l'Ouest ont amplifié ces différences » (p. 3). S'il ne fait aucun doute qu'il y a eu des différences avec les pays occidentaux, chercher à mesurer les écarts s'avère certainement hasardeux. Car d'aucuns pourraient tout aussi bien objecter leur atténuation en convoquant, par exemple, la mise en place des États-providence en Europe de l'Ouest après la Seconde Guerre mondiale - on se rappellera alors que William Beveridge a en partie tiré la matière de son fameux rapport de ses échanges avec Beatrice et Sidney Webb qui avaient été très impressionnés par ce qu'ils avaient vu en Union soviétique en 1932.

1 - Dominique Pestre, « Pour une histoire sociale et culturelle des sciences. Nouvelles définitions, nouveaux objets, nouvelles pratiques ", Annales. Histoire, Sciences Sociales, 3 (1995), p. 487-522.

2 - Michel Espagne, Les transferts culturels franco-allemands, P. : PUF, 1999.

3 - Kapil Raj, Relocating Modern Science : Circulation and the Construction of Knowledge in South Asia and Europe, 1650-1900, Houndmills : Palgrave Macmillan, 2007.

\section{Gregory Dufaud}

IEP, Lyon 